\title{
Polar ordering of quasiliquid crystals-An optical second harmonic generation study
}

\author{
H. Hsiung, Th. Rasing, and Y.R. Shen \\ Department of Physics, University of California, Berkeley, California 94720 \\ F. P. Shvartsman, ${ }^{\text {a) }}$ I. R. Cabrera, and V. A. Krongauz \\ Department of Structural Chemistry, The Weizmann Institute of Science, Rehovot 76100, Israel
}

(Received 10 April 1987; accepted 28 May 1987)

\begin{abstract}
Supercooled quasiliquid crystal films are studied by optical second harmonic generation. The experimental results indicate that a polar ordering of molecules exists in the absence of external fields. By applying an electrostatic field, the polar ordering can be modified in a manner predictable by a mean-field theory.
\end{abstract}

\section{INTRODUCTION}

A quasiliquid crystal ${ }^{1,2}$ (QLC) is an intrinsic two-component medium consisting of spiropyrans in equilibrium with their merocyanine counterparts through the following thermo- or photochromic reaction:

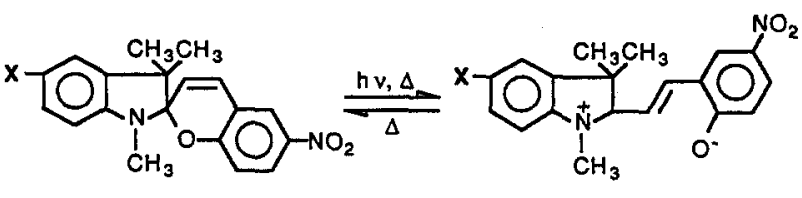

SPIROPYRAN

MEROCYANINE

where the mesogenic group $\mathbf{X} \equiv \mathbf{R P h C O O P h C H}=\mathbf{N}-$ ( $\mathrm{R} \equiv \mathrm{CH}_{3} \mathrm{O}-, \mathrm{C}_{6} \mathrm{H}_{13} \mathrm{O}$-, or $\mathrm{CN}-$ ) is responsible for inducing a nematic-like ordering over a wide temperature range. Though the equilibrium concentration of the merocyanines is low $(3 \%-10 \%)$, the presence of these highly polar molecules nonetheless plays a major role in stabilizing (against crystallization) the metastable QLC state, which occurs well below the melting point of the crystalline spiropyran. QLC films aligned under an electrostatic field $(>5 \mathrm{kV} / \mathrm{cm}) d u r$ ing preparation have shown enhanced stability; such films can be supercooled with their molecular ordering preserved at room temperature over many months.

Various techniques [miscibility, $\mathrm{x}$-ray diffraction, electron spin resonance (ESR), UV-visible and FT-IR spectroscopies] have been used to study the structure of (supercooled) QLC films. ${ }^{1}$ It has been established that both the spiropyrans and merocyanines exhibit long-range orientational ordering with their order parameters $S$ (determined by dichroism or ESR) roughly equal to 0.4 . Remained unanswered was the question whether the QLC state is centrosymmetric, as the conventional nematic state, or noncentrosymmetric with a ferroelectric-type arrangement. Optical second harmonic generation ${ }^{3}$ (SHG) is an ideal tool for such a study because within the electric-dipole approximation, the second-order nonlinear process is forbidden in a centrosymmetric medium.

We report here a SHG study of supercooled QLC films. The results indicate that $Q L C$ is noncentrosymmetric with a polar axis pointing along the director (i.e., the average mo-

\footnotetext{
a) Current address: Imaging Systems Department, Experimental Station, E. I. Du Pont de Nemours \& Co., Wilmington, DE 19898.
}

lecular orientation ) - defined by the direction of the aligning electrostatic field during preparation. This symmetry should arise from a polar alignment of the molecular dipole moments. The polar ordering of molecules can be modified by an external electrostatic field along the director, as manifested by the $E$-field dependence of the SHG.

In Sec. II, we present a mean-field theory for the SHG from QLC films. This is followed by a description of the experimental arrangement (Sec. III) and experimental results (Sec. IV). A discussion of our observations is given in Sec. V.

\section{THEORY}

A nonlinear polarization $\mathbf{P}^{\mathrm{NL}}$, of frequency $2 \sigma$, can be induced in a medium irradiated by a laser field $\mathbf{E}$, of frequency $\varpi$, as described by the relation

$$
\mathbf{P}^{\mathrm{NL}}(2 \sigma)=\chi^{(2)}(2 \sigma=\sigma+\varpi): \mathbf{E}(\varpi) \mathbf{E}(\varpi) .
$$

$\mathbf{P}^{\mathrm{NL}}$ then acts as a source that radiates coherent waves at $2 \sigma$. The nonlinear susceptibility $\chi^{(2)}$ is a third-rank tensor which reflects both the symmetry and the material properties of the medium. For a centrosymmetric medium, for example, $\chi^{(2)}$ is identically zero under the electric-dipole approximation; while for a medium exhibiting complete rotational symmetry about a polar axis $\hat{\mathbf{x}}$ (i.e., the $\mathbf{C}_{\infty v}$ symmetry), the nonzero elements of $\chi^{(2)}$ are $\chi_{x x x}^{(2)}, \chi_{x y y}^{(2)}=\chi_{x z z}^{(2)}$, and $\chi_{y y x}^{(2)}=\chi_{y x y}^{(2)}=\chi_{z z x}^{(2)}=\chi_{z x z}^{(2)}$. In an organic substance where the identities of individual molecules are preserved, the macroscopic $\chi^{(2)}$ can be related to the molecular hyperpolarizability $\beta(2 \varpi=\varpi+\varpi)$ through a statistical averaging over molecular orientations:

$$
\chi^{(2)}=N\langle\boldsymbol{\beta}: \mathbf{L}(2 \sigma) \mathbf{L}(\varpi) \mathbf{L}(\varpi)\rangle,
$$

where $N$ is the number density and the L's are the local-field correction factors ${ }^{4}$ which account for the molecular interactions in the substance. In our later analysis, we shall use the well-known Lorenz-Lorentz form, $\mathbf{L}(\boldsymbol{\sigma})=[\epsilon(\boldsymbol{\sigma})+2] / 3$, $\epsilon$ being the linear dielectric tensor of the medium.

Since merocyanine dyes are known to have very large molecular hyperpolarizabilities $\left.\left(\beta \sim 10^{-28}-10^{-27} \mathrm{esu}\right)\right)^{5}$ we can assume that SHG in QLCs is dominated by the merocyanine molecules. For simplicity, we further assume that both $\beta$ and the permanent dipole moment $p$ of merocyanines are dominated by a single component along the long molecu- 
lar axis $(\hat{\xi})$, that is, by $\beta_{\xi \xi \xi}(\equiv \beta)$ and $p_{\xi}$ ( $\left.\equiv p\right)$, respectively. The validity of these assumptions will be discussed later. We then obtain, from Eq. (3),

$\chi_{x x x}^{(2)}=N \beta L_{\mathrm{x}}(2 \sigma)\left[L_{x}(\varpi)\right]^{2}\left\langle\cos ^{3} \theta\right\rangle$,

$\chi_{x y y}^{(2)}=N \beta L_{\mathrm{x}}(2 \varpi)\left[L_{y}(\varpi)\right]^{2}\left[\langle\cos \theta\rangle-\left\langle\cos ^{3} \theta\right\rangle\right] / 2$,

$\chi_{y y x}^{(2)}=N \beta L_{y}(2 \varpi) L_{y}(\varpi) L_{x}(\varpi)\left[\langle\cos \theta\rangle-\left\langle\cos ^{3} \theta\right\rangle\right] / 2$,

where $\theta$ is the angle between $\hat{\xi}$ and $\hat{\mathbf{x}}$, and $N$ now refers to the number density of the merocyanines. For the averaging over molecular orientations, we assume the "mean field" potential experienced by an individual merocyanine molecule in a QLC in the presence of an external dc field, $\mathbf{E}_{0}(=E \hat{\mathbf{x}})$, to be

$$
\begin{aligned}
& V(\theta)=V_{N}(\theta)-u \cos \theta-\mathbf{p} \cdot \mathbf{L}_{0} \mathbf{E}_{0}, \\
& V_{N}(\theta)=-u_{2} P_{2}(\cos \theta)-u_{4} P_{4}(\cos \theta)-\cdots,
\end{aligned}
$$

where $\mathbf{L}_{0} \equiv \mathbf{L}(\varpi=0), P_{n}$ is the $n$ th-order Legendre polynomial, and $V_{N}$ is the conventional nematic mean-field potential. ${ }^{6}$ The coefficients $u$ and $u_{n}$ are determined by molecular ordering and interaction strength. The term $u \cos \theta$ is included here to account for the possible polar symmetry of QLCs even in the absence of an external dc field. Both this term and the $p \cdot L_{0} \mathbf{E}_{0}$ term will be considered as perturbations to the nematic potential in the present case. The orientationaveraged value of an arbitrary function $f(\theta)$ can then be obtained from

$$
\begin{aligned}
\langle f(\theta)\rangle= & \int_{-1}^{1} f(\theta) \exp [-V(\theta) / k T] d(\cos \theta) / \\
& \int_{-1}^{1} \exp [-V(\theta) / k T] d(\cos \theta) \\
\approx & \langle f(\theta)\rangle_{0}+\langle f(\theta) \cos \theta\rangle_{0}\left(u+p L_{0} E\right) / k T
\end{aligned}
$$

where

$$
\begin{aligned}
\langle g(\theta)\rangle_{0} \equiv & \int_{-1}^{1} g(\theta) \exp \left[-V_{N}(\theta) / k T\right] d(\cos \theta) / \\
& \int_{-1}^{1} \exp \left[-V_{N}(\theta) / k T\right] d(\cos \theta) .
\end{aligned}
$$

Since $V_{N}(\theta)$ is an even function of $\cos \theta$, we have

$$
\begin{aligned}
\left\langle\cos ^{n} \theta\right\rangle & =\left\langle\cos ^{n} \theta\right\rangle_{0} & & \text { for even } n, \\
& =\left\langle\cos ^{n+1} \theta\right\rangle_{0}\left(u+p L_{0} E\right) / k T & & \text { for odd } n .
\end{aligned}
$$

From Eqs. (4) and (7), we notice that $\chi^{(2)}$ should depend linearly on $E$, while the ratio between any two $\chi_{i j k}^{(2)}$ 's should remain constant. On the other hand, the nematic-type order parameter

$$
S \equiv\left\langle P_{2}(\cos \theta)\right\rangle \equiv\left(3\left\langle\cos ^{2} \theta\right\rangle-1\right) / 2=\left\langle P_{2}(\cos \theta)\right\rangle_{0}
$$

should be independent of the $E$ field.

\section{EXPERIMENTAL ARRANGEMENT}

We prepared the samples by first putting a small amount of a spiropyran-benzene solution on preheated fused-silica slides (at $\sim 80^{\circ} \mathrm{C}$ ), and QLC films were then formed on the surface of the slides after the evaporation of the solvent. A
3:1 mixture of spiropyrans with $\mathrm{R} \equiv \mathrm{CH}_{3} \mathrm{O}$ - and $\mathrm{C}_{6} \mathrm{H}_{13} \mathrm{O}$ was used in the solution, which yields a QLC phase in the temperature range $50-135^{\circ} \mathrm{C} .{ }^{2}$ Single-domain, planar (i.e., director lying in the surface plane) films were prepared by applying an electrostatic field parallel to the surface during the film-forming process. The field was produced by applying a voltage across a pair of parallel aluminum thin-film electrodes which had been previously deposited on the slide. In our experiment, $2.5 \mathrm{kV}$ was applied to the electrodes separated by $2 \mathrm{~mm}$. After the QLC films were formed, they were quickly cooled down to the room temperature and the aligning field was then removed. The thickness of these films was controlled by the amount of the spiropyran solution used in the preparation; our sample thickness was $\sim 1-3 \mu \mathrm{m}$.

For the SHG experiment, we used excitation laser pulses at $1.06 \mu \mathrm{m}$ generated by a $Q$-switched Nd:YAG laser. Each pulse had a duration of $10 \mathrm{~ns}$ and an energy limited to $<2 \mathrm{~mJ}$ to avoid damaging the QLC films. The laser beam was nearly normally incident and focused to a beam diameter of $\sim 0.6 \mathrm{~mm}$ at the sample. The second-harmonic ( $\mathrm{SH}$ ) photons at $532 \mathrm{~nm}$ were detected in the reflected direction. ${ }^{7}$ Since the SH frequency falls within the first electronic excitation band (centered around $600 \mathrm{~nm})^{2}$ of the merocyanines, $\beta$ of these molecules is expected to be resonantly enhanced. Due to the nature of the sample preparation, the thickness of a QLC film is usually nonuniform, and the QLC-air interface is not flat. It was, therefore, best to excite the QLC film from the substrate side, and detect SH reflection from the flat QLC-substrate interface. A spatial filter in the detection path could eliminate most of the SH photons scattered from the QLC-air interface. The advantage of such an experimental geometry is twofold: First, the effective coherence length of SH reflection is only about a reduced wavelength of light, ${ }^{3}$ hence the SHG is independent of the film thickness. Therefore, no precise measurements of the thickness and the optical constants are needed. Second, normal incidence of light eliminates the complication of multipole (e.g., electric-quadrupole, magnetic-dipole) contributions to the SHG, which generally exist in centrosymmetric media. ${ }^{8}$

Components of $\chi^{(2)}$ were determined by using different combinations of the polarization of the excitation waves and that of the SH waves. The absolute values of $\chi_{i j k}^{(2)}$ 's were deduced from the intensities of the SH signal by the formalism given in Ref. 3, and were calibrated against a reference quartz crystal. The dc-field dependence of $\chi^{(2)}$ was also studied using a variable electrostatic field along the director. A simultaneous measurement of the optical phase retardation,

$$
\Delta \phi=2 \pi(d / \lambda) \Delta n
$$

( $d$-film thickness; $\lambda$-wavelength of a probing laser), was performed to monitor the optical birefringence $\Delta n$ of the QLC in the presence of the dc field. This was accomplished by a polarization-modulation technique ${ }^{9}$ using a He-Ne la$\operatorname{ser}(\lambda=632.8 \mathrm{~nm})$; the resolution in $\Delta \phi$ was $\sim 4 \times 10^{-4} \mathrm{rad}$ in our measurement. The birefringence $\Delta n$ can be regarded as a macroscopic orientational order parameter which is roughly proportional to the microscopic order parameter $S$ defined in Eq. (8). ${ }^{10}$ 


\section{EXPERIMENTAL RESULTS}

Under the experimental arrangement with the director and the $E$ field both along the $\hat{x}$ direction, the QLC film in the $X Y$ plane, and the waves propagating in the $\pm \hat{\mathbf{z}}$ directions, we could measure independently six components of $\chi^{(2)}-\chi_{x x x}^{(2)}, \chi_{x y y}^{(2)}, \chi_{y y x}^{(2)}=\chi_{y x y}^{(2)}, \chi_{y x x}^{(2)}$, and $\chi_{y y y}^{(2)}$. We found that the last two components vanish with or without the dc $E$ field, a property consistent with the $\mathbf{C}_{\infty v}$ symmetry. Other components all showed linear dependence on $E$, with the ratio between any two components being constant, as illustrated in Fig. 1. We obtained $\chi_{y y x}^{(2)} / \chi_{x x x}^{(2)}$ $\approx 0.15, \chi_{x y y}^{(2)} / \chi_{x x x}^{(2)} \approx 0.09$. Within our accuracy, the "compensation field," $E_{c}(\approx-4 \mathrm{kV} / \mathrm{cm})$, to make $\chi_{i j k}^{(2)}\left(E_{c}\right)=0$ is the same for all the three components. In general, $\chi_{i j k}^{(2)}$ 's are complex numbers, and their phases cannot be determined by the measurement of SH intensities alone. However, in light of Eq. (4), the relative phases of these quantities should be the same, and, as indicated by Eq. (7), a change of phase by $\pi$ may occur following the change of the polarity of the "effective" $E$ field ( $\equiv E-E_{c}$ ). In Fig. 1, therefore, the $\chi_{i j k}^{(2)}$ 's are taken to be real numbers with an imposed change of sign after their values drop to zero. Within the same QLC film, the variation in the value of a component $\chi_{i j k}^{(2)}$ was $\sim \pm 10 \%$. From sample to sample, however, variation by roughly a factor of 3 has been observed, presumably because of variations in the merocyanine concentration and molecular orientational order. For a typical sample represented by Fig. $1, \chi_{x x x}^{(2)}(E=0) \approx 8.0 \times 10^{-11}$ esu. Samples with positive

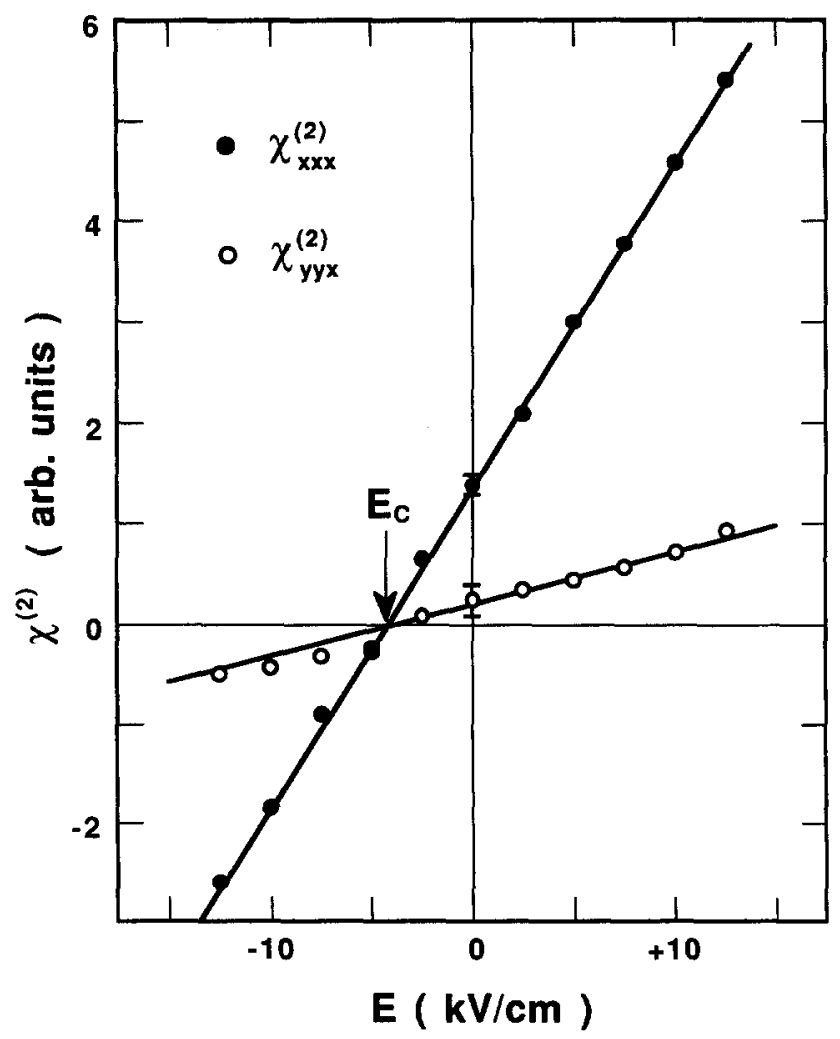

FIG. 1. Two tensor components of the nonlinear susceptibility $\chi^{(2)}$ as functions of the strength of an electrostatic field applied along the director $(\hat{\mathbf{x}})$ of a QLC film.
$E_{c}$ [i.e., $\chi_{i j k}^{(2)}(E=0)$ being negative relative to Fig. 1] have also been observed, though the linear dependence on $E$ was not strictly followed, especially for smaller $E$ fields, by these samples. No observable change in the phase retardation $\Delta \phi$ was found in QLC films as the $E$ field was applied, indicating $\Delta n$ to be independent of $E$, as expected from Eq. (8).

SHG signal (without the dc $E$ field) from fresh QLC films was found to first decrease monotonously during the initial hours after the films were prepared, then settle down and remain essentially unchanged after about 1-2 days. The corresponding decrease in $\chi_{x x x}^{(2)}(E=0)$ was usually a factor of 2 to 3 . This effect is presumably due to relaxation of molecular orientations in QLC towards a metastable orientational distribution. All the results described previously in this section were obtained from samples aged for one to two months.

\section{DISCUSSION}

In the absence of external fields, the nonvanishing $\chi^{(2)}$ indicates that the QLC is noncentrosymmetric. The $E$ field dependence of $\chi_{i j k}^{(2)}$ 's, shown in Fig. 1, agrees with Eqs. (4) and (7) if we let $u=-p L_{0} E_{c}$. We further check the quantitative agreement with the theory as follows: From the experimental values of $\chi_{y y x}^{(2)} / \chi_{x x x}^{(2)}$ and $\left\langle\cos ^{2} \theta\right\rangle_{0}=(2 S+1) / 3$, with $S \approx 0.4$, we could deduce from Eqs. (4) and (7) that $\left\langle\cos ^{4} \theta\right\rangle_{0} \approx 0.45$, i.e., $\left\langle P_{4}(\cos \theta)\right\rangle \approx 0.09$, a reasonable value for the nematic ordering. ${ }^{11}$ Taking the number density $N$ of merocyanines to be $\sim 2 \times 10^{19} \mathrm{~cm}^{-3}$ ( $\sim 5 \%$ of the total density $)^{12}$ and their molecular dipole moment $p \sim 10 \mathrm{D},{ }^{13}$ the optical dielectric constants $\epsilon_{x} \sim 2.6$ and $\epsilon_{y} \sim 2.3$ (neglecting optical dispersion), the dc dielectric constant $\epsilon_{x} \sim 10-20$, and using the experimental values of $\chi_{x x x}^{(2)}$ and $E_{\mathrm{c}}$, we obtain from Eq. (4) that $\beta \sim(1-2) \times 10^{-28}$ esu for the QLC merocyanines. This value is of the same order of magnitude as those of other merocyanines. ${ }^{5}$ The consistency of the theory is also checked by the $E$ field dependence of the order parameter $S$ : our result shows that $\Delta \phi(\propto S)$ is independent of $E$, in agreement with the prediction of Eq. (8).

The experimental value of $\chi_{x y y}^{(2)} / \chi_{y y x}^{(2)}$ is $\approx 0.6$. From Eq. (4), this suggests that $L_{x}(2 \varpi) L_{y}(\varpi) / L_{y}(2 \varpi) L_{x}(\varpi) \sim 0.6$. This could be the result of local-field dispersion due to a resonance band near $2 \varpi$ in merocyanines. On the other hand, it may also be the consequence of our simplifying assumption that all but one component of $\beta$ are negligible. Spiropyrans, because of their much larger concentration in QLCs, may also contribute to the SHG even though their $\beta$ and $p$ are much smaller than those of the merocyanines. Such a correction, however, can be easily included in the theory. The opposite polarities of $\chi^{(2)}(E=0)$ (or $E_{c}$ ) observed in different samples should correspond to the two metastable states of the polar ordering with their symmetry axes pointing in the $\pm \hat{\mathbf{x}}$ directions, respectively. The nonlinear dependence on $E$ in the case of "negative $\chi^{(2)}(E=0)$ " indicates that the two states are nondegenerate; the negative $\chi^{(2)}(E=0)$ state is less stable and more easily influenced by the applied field.

It is possible that in addition to molecular reorientation, a pure electronic process described by 
$\mathbf{P}^{\mathrm{NL}}(2 \sigma)=\chi^{(3)}(2 \sigma=0+\sigma+\varpi): \mathbf{E}_{0} \mathbf{E}(\varpi) \mathbf{E}(\varpi)$

may also contribute to the field-induced part of the SHG. However, for this contribution to be important, $\chi^{(3)}$ in Eq. (10) must have a value $\sim(2-6) \times 10^{-12}$ esu-which seems to be too large for typical molecules. Therefore, the pure electronic contribution is not likely to be very significant.

Polar ordering has never been observed in conventional liquid crystals in the nematic state; that is, molecules tend to have equal probabilities pointing in opposite directions along the director. Local antiferroelectric pairing of molecules is also known to exist in many LCs consisting of strong polar molecules. This is due to a tendency of nearby dipoles preferring to be antiparallel to each other in order to reduce their dipole-dipole interactions. ${ }^{14}$ In the QLCs, however, nearby merocyanine molecules are separated by a few spiropyrans, which could screen the dipole-dipole interactions among merocyanines and allow a preferred polar alignment of these molecules; in other words, the spiropyrans are responsible for the interaction potential $-u \cos \theta$ given in $\mathrm{Eq}$. (5). The spiropyrans themselves could also have a polar ordering with an axis presumably pointing opposite to that of the merocyanines.

In conclusion, using the SHG technique, we have studied the polar ordering of supercooled QLC films, and the influence of an electrostatic field on such an ordering. The results were interpreted in terms of a mean-field theory. The current work can be extended to the study of the polar ordering at temperatures across the QLC phase, as well as during the formation of QLC films.

\section{ACKNOWLEDGMENTS}

Helpful discussions with Dr. G. R. Meredith are appreciated by the authors. This work was supported by a research grant from E. I. du Pont de Nemours \& Co., including a research associateship for one of us (H.H.). V. K. acknowledges support from the U. S. A. Army European Research Office and Minerva Foundation, FRG.

'F. P. Shvartsman, I. R. Cabrera, A. L. Weiz, E. J. Wachtel, and V. A Krongauz, J. Phys. Chem. 89, 3941 (1985), and references therein.

${ }^{2}$ F. P. Shvartsman and V. A. Krongauz, Nature 309, 608 (1984).

${ }^{3}$ See, for example, Y. R. Shen, The Principles of Nonlinear Optics (Wiley, New York, 1984), Chaps. 6 and 7.

${ }^{4}$ See, for example, N. Bloembergen, Nonlinear Optics (Benjamin, New York, 1965), p. 69.

${ }^{5}$ D. J. Williams, Angew. Chem. Int. Ed. Engl. 23, 690 (1984).

${ }^{6}$ See, for example, E. B. Priestley, P. J. Wojtowicz, and P. Sheng, Introduction to Liquid Crystals (Plenum, New York, 1975), Chap. 4.

${ }^{7}$ The details of the experimental technique and arrangement can be found in the review articles by Y. R. Shen, Annu. Rev. Mater. Sci. 16, 69 (1986); and in New Laser and Optical Investigations of Chemistry and Structure at Interfaces, edited by R. B. Hall and A. B. Ellis (Verlag-Chemie, Berlin, 1986), p. 151.

${ }^{8}$ P. Guyot-Sionnest, W. Chen, and Y. R. Shen, Phys. Rev. B 33, 8254 (1986).

${ }^{9}$ G. Maret and K. Dransfeld, Physica (Utrecht) 86-88 B+C, 1077 (1977).

${ }^{10}$ R. G. Horn, J. Phys. (Paris) 39, 105 (1978), and references therein.

"S. D. Durbin and Y. R. Shen, Phys. Rev. A 30, 1419 (1984), and references therein.

${ }^{12}$ The total density was estimated from the average volume occupied by a single molecule (molecular length $\sim 28 \AA \AA$; separation between molecules $\sim 9 \AA$ A , from Ref. 1$)$.

${ }^{13}$ E. D. Bergmann, A. Weizmann, and E. Fischer, J. Am. Chem. Soc. 72, 5009 (1950).

${ }^{14}$ See, for example, S. Chandrasekhar, Liquid Crystals (Cambridge University, Cambridge, 1977), p. 83. 